\title{
Evaluating MDC with Incentives in P2PTV Systems*
}

\author{
Alberto J. Gonzalez ${ }^{1,2}$, Andre Rios ${ }^{1,2}$, Guillermo Enero ${ }^{2}$, \\ Antoni Oller ${ }^{1,2}$, and Jesus Alcober ${ }^{1,2}$ \\ ${ }^{1}$ Dept. of Telematics Engineering, Universitat Politecnica de Catalunya (UPC) \\ Barcelona, Spain \\ 2 i2Cat Foundation, Barcelona, Spain \\ \{alberto.jose.gonzalez, andre.rios, antoni.oller, jesus.alcober \\ a $\{$ upc.edu, i2cat.net $\}$, \\ guillermo.enero@i2cat.net
}

\begin{abstract}
The popularity of $\mathrm{P} 2 \mathrm{P}$ video streaming is raising the interest of broadcasters, operators and service providers. Concretely, mesh-pull based P2P systems are the most extended ones. Despite these systems address scalability efficiently, they still present limitations that difficult them to offer the same user experience in comparison with traditional TV. These ones are mainly the freeriding effect, long start-up delays and the impact of churn and bandwidth heterogeneity. In this paper we study the performance of Multiple Description Coding (MDC) combined with the use of incentives for redistribution in order to mitigate some of them by means of simulations. Simulation results show that the use of MDC and incentive-based scheduling strategies improve the overall performance of the system. Moreover, an extended version of the P2PTVSim simulator has been developed to support MDC and incentives.
\end{abstract}

Keywords: P2P Streaming, MDC, Incentives, Continuity Index (CI), Delay.

\section{Introduction}

P2PTV streaming systems have become a popular service on the Internet (both at commercial and research level), with several successful deployments [1], and the most spread form of what is known as Internet TV. The use of these systems is promising because they offer the possibility to introduce added value to traditional TV broadcasting by providing flexibility, in terms of content delivery and interactive services. However, in order to become a truly successful application they need to be able to provide the same or even better user experience as TV broadcasting offers. P2PTV systems are expected to provide a high degree of scalability with different streaming rates and number of peers. They must also provide continuity under adverse churn conditions (especially in presence of flash-crowds) as well as ensuring delivery of data within a given deadline in order to provide smooth playback. Some aspects affecting the performance of these requirements are: free-riding (non-cooperation)

\footnotetext{
*This work was supported by MCyT (Spanish Ministry of Science and Technology) under the Project TSI2007-66637-C02-01, which is partially funded by FEDER.
} 
effect, long start-up delay and the impact of churn and bandwidth heterogeneity in the stability of the system. We tackle them in this work.

In this paper we propose an MDC-based system, which uses incentives for redistribution, in order to address the impact of losses in the Continuity Index (CI), delay and the performance problems due to the effect of free-riding. MDC is a technique designed to enhance error resilience and increase transmission robustness and scalability by means of splitting a stream into $\mathrm{N}$ different sub-streams $(\mathrm{N} \geq 2)$. Different splitting techniques can be used [2].

In order to validate the proposed solution we have deployed it in a simulatior called P2PTVSim [3]. However, we have extended it in order to be able to simulate the usage of MDC as well as the use of a specific incentive strategy (inspired by [4]). The obtained results show how the use of MDC provides a more robust behaviour against losses. Consequently, the Continuity Index of the system is improved. In addition, thanks to the use of incentives, the effect of free-riding is alleviated.

\section{Proposed Solution}

The simulated system is a mesh-pull-based P2P streaming system that uses MDC for providing robustness to the communication. In order to support MDC, the receiverside scheduler was adapted. When the system uses MDC, four balanced descriptions are generated and distributed in chunks. It also introduces the use of incentives based on the contribution of the partners in the supplier-side scheduler. Incentives are used in order to encourage cooperation, so that those peers contributing more to their partners are more likely to receive more descriptions and therefore more quality.

\subsection{Receiver-Side Scheduler}

The Buffer Map consists of a matrix with as many rows as descriptors. Partners exchange their Buffer Maps periodically and they perform rounds of chunk requests to get the missing chunks considering the availability information provided by their neighbours. The schedule of these requests is critical to achieve an optimal result, retrieve the maximum number of descriptions and ensure the best CI and quality. Chunks that have already been received are marked with a $\mathrm{B}$ and chunk that have been requested with $\mathrm{R}$. In the matrix, each chunk position has a number indicating the order of the requests. The scheduler looks, at the beginning of each round, for the first chunk index that does not have a buffered (B) or requested $(\mathrm{R})$ chunk from any description. When it finds the first chunk index satisfying this condition it selects a random description (from the available ones according to the availability information provided by its neighbours) and makes the request. Then, it continues with the next chunk index that has non-buffered or requested chunk until there are no more chunk indexes fulfilling this condition. The scheduling algorithm continues by doing the same routine over chunk indexes with just one buffered or requested chunk, then with just two, and so on. The goal of this scheduling algorithm is to get the maximum number of descriptions for each chunk index time but always trying to avoid having high variation between the received number of descriptions from one time to another. 


\subsection{Supplier-Side Incentive-Based Scheduler}

The supplier-side algorithm takes into account the contribution of their partners and serves them accordingly (incentivates). The effect of this supplier strategy is that peers that contribute more are more likely to receive a larger number of descriptions. As a supplier, each peer has a queue of requests from its partners and at each round it has to decide which of these requests is going to be served first. Instead of selecting a random one or handle them in a FIFO manner, a weighted selection is performed. The weight in this case is assigned by computing the percentage of chunks that have been provided by a specific neighbour from the total.

\section{Simulation Results}

Four different simulation scenarios were considered. The first scenario is the reference system, which is a mesh-pull P2P streaming system with single layered video and no incentives (default operation in P2PTVSim). Then, in a second scenario, we added incentives. The third one is an MDC-based (using 4 layers) mesh-pull P2P streaming system and the last scenario is a variation of the third one, adding incentives. Due to space limitations, we focus on the first and the last scenarios.

The parameters for the simulations are the following ones: a total of 1000 peers, with a mean degree of 10 partners and a 5 seconds buffer. Four different types of peers: class $\mathrm{A}$, peers with $5 \mathrm{Mbps}$ of upload bandwidth (10\% of the total); class B, peers with $1 \mathrm{Mbps}$ of upload bandwidth ( $40 \%$ of the total); class C, peers with $500 \mathrm{Kbps}$ of upload bandwidth (40\% of the total); and class D, peers that act as freeriders, with $0 \mathrm{Kbps}$ of upload bandwidth (10\% of the total). No download bandwidth constraint is assumed. The simulations last for the distribution of 2500 chunks and the chunk size was $1 \mathrm{~KB}$. For each scenario several simulations have been performed varying the packet loss rate according to a random loss model (Bernoulli).

The metrics that are measured for the evaluation of the techniques are the end-toend delay, the $\mathrm{CI}$ and the average number of received descriptions.

\subsection{Reference System}

When considering the effect of losses (Fig. 1) in the reference system we can see that the CI decreases as the losses increase. The delay, as it can be seen in Fig. 2, increases as the percentage of losses increases and it is in the range of $1,5 \mathrm{~s}$ to $3,5 \mathrm{~s}$.

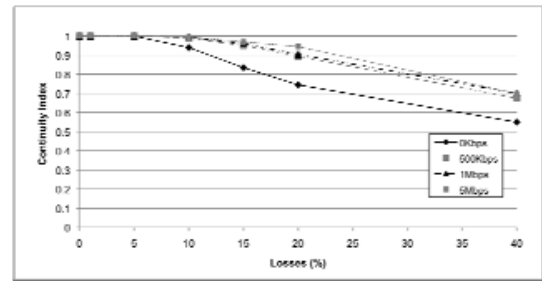

Fig. 1. Reference system - CI vs. losses

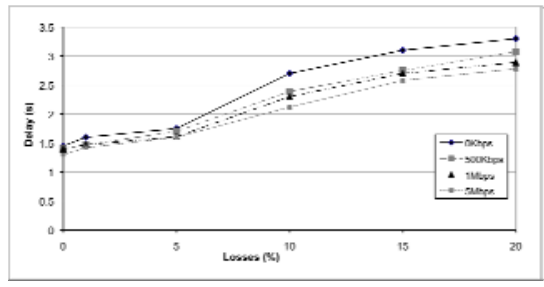

Fig. 2. Reference system - Delay vs. losses 


\subsection{System with Incentives}

Finally, we combined the use of MDC with incentives. Here, to the improvements introduced by MDC in terms of CI increase and low end-to-end delay we can add the overall performance boost introduced by the use of incentives. As Fig. 3 shows, the CI can be maintained at almost the maximum level even at high loss rates (40\%). This combination allows a high level of playback continuity. The quality, in terms of average number of descriptions is also increased for the cooperating peers. Regarding to delay (Fig. 4) it is approximately the same delay that the MDC system provides without incentives.

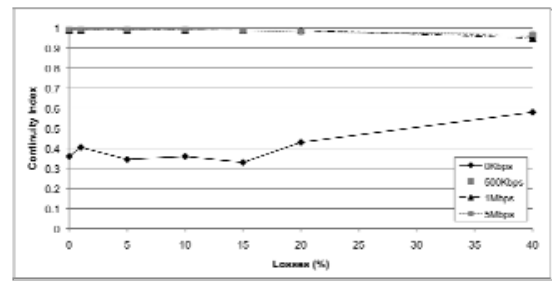

Fig. 3. MDC+incentives - CI vs. Losses

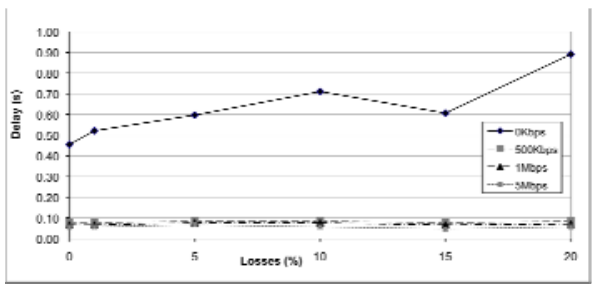

Fig. 4. MDC+incentives - Delay vs. Losses

\section{Conclusions and Future Work}

The aim of this work was to study the performance of MDC systems with incentive mechanisms in P2PTV systems. The evaluation of the solution was done by means of simulation. The gathered results show that the proposed solution clearly improves the considered metrics and the overall behaviour of the system compared to the performance of the reference system simulating a common P2PTV system. These results can be used as reference or guideline for further developments. As an additional outcome of this work, we have developed a simulation software (based on P2PTVSim) which provides a valid test-bed that can be used for future studies. Future work will include an analysis of the overhead introduced by MDC, PSNR measurement for quality estimation and the simulation considering churn effect. Start-up delay reduction will also be studied by means of MDC.

\section{References}

1. Xiaojun, H., Chao, L., Jian, L., Yong, L., Ross, K.: A Measurement Study of a Large-Scale P2P IPTV System. IEEE Transactions on Multimedia 9(8), 1672-1687 (2007)

2. Tillier, C., Crave, O., Pesquet-Popescu, B., Guillemot, C.: A Comparison of four Video Multiple Description Coding Schemes. In: EUSIPCO, Poznan (2007)

3. P2PTVSIM, http://www. napa-wine.eu/cgi-bin/twiki/view/Public/P2PTVSim

4. Zhengye, L., Yanming, S., Panwar, S.S., Ross, K.W., Wang, Y.: P2P Video Live Streaming with MDC: Providing Incentives for Redistribution. In: IEEE International Conference on Multimedia and Expo., pp. 48-51, 2-5 (2007) 